\title{
¿Tienes lo que hay que tener?: Un análisis de los factores asociados con la intención de optar por una carrera en cirugía entre estudiantes de medicina
}

\author{
Luis Carlos Domínguez T. ${ }^{1}$, Diego Sierra B. ${ }^{1}$, Valentín Vega P. ${ }^{1}$, \\ Laura Gómez A. ${ }^{1}$, Maikel Pacheco C. ${ }^{2}$ y Felipe Vargas B. ${ }^{3}$
}

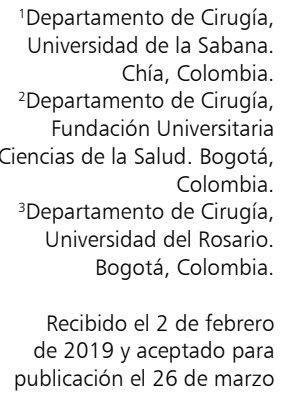

publicación el 26 de marzo de 2019.

Correspondencia a: Dr. Luis Carlos Domínguez T. carlosdot@unisabana.edu.co
Do you have what it takes? An analysis of the factors associated with the intention to choose a surgical career among medical undergraduates

Introduction: The changes in the professional practice have transformed the intention of medical students to choose a surgical career. Aim: Evaluate the factors associated with this intention in a context of accelerated educational and health dynamics. Materials and Method: We evaluated the intention of the students of ten medical schools in Colombia, and the associated factors, through a questionnaire (Likert scale) settled from a literature review. We identify the association between the factors and the intention by using linear regression analysis. The results of the regression are presented by $\beta$ coefficients $(p<0.05)$. Results: A total of 252 students ( $65.87 \%$ women) were included in the analysis. The average of the intention was $3.15 \pm 1.34(1-5)$. The factors significantly associated were: identification with the surgical lifestyle $(\beta=0.25)$; identification with models $(\beta=0.18)$; personal attributes $(\beta=0.25)$ and identification with the professional practice of the surgeon $(\beta=0.16)$. We did not find significant associations with gender (women in the specialty), prestige, postgraduate dynamics and future rewards. Conclusion: There is a moderate intention to choose a career in surgery among Colombian medical undergraduates. Choosing this career requires personal attributes, models and identification with the lifestyle and professional practice of the surgeon. Further qualitative studies are necessary to explore these results in depth, as well as interventions to stimulate this intention among students.

Key words: professional career; surgery; intention; medical students; Colombia.

\section{Resumen}

Introducción: Los cambios en la práctica profesional han transformado la intención de los estudiantes de medicina de optar por una carrera en cirugía. Objetivo: Evaluar los factores asociados con esta intención en un contexto de acelerada dinámica educativa y sanitaria. Materiales y Método: Evaluamos la intención de los estudiantes de diez facultades de medicina en Colombia, y los factores asociados, mediante un cuestionario (escala de Likert) desarrollado a partir de la revisión de la literatura. Identificamos la asociación entre los factores y la intención mediante un análisis de regresión lineal. Los resultados de la regresión se presentan mediante coeficientes $\beta$ ( $p<0,05)$. Resultados: Un total de 252 estudiantes $(65,87 \%$ mujeres $)$ fueron incluidos en el análisis. El promedio de la intención fue 3,15 $\pm 1,34$ (1-5). Los factores significativamente asociados fueron: identificación con el estilo de vida quirúrgico $(\beta=0,25)$; identificación con modelos $(\beta=0,18)$; atributos personales $(\beta=0,25)$ e identificación con la práctica profesional del cirujano $(\beta=0,16)$. No encontramos asociación significativa con el género (mujeres en la especialidad), prestigio, dinámica del posgrado ni recompensa futura. Conclusión: Existe una intención moderada de elegir una carrera de cirugía entre estudiantes de medicina colombianos. Optar por esta carrera requiere atributos personales, modelos e identificación con el estilo de vida y práctica profesional del cirujano. Se requieren estudios cualitativos que exploren en profundidad estos resultados e intervenciones focalizadas a estimular esta intención entre los estudiantes.

Palabras clave: carrera profesional; cirugía; intención; estudiantes de medicina; Colombia. 


\section{Introducción}

Uno de los seis indicadores de la Comisión Lancet en Cirugía Global para el monitoreo del acceso a cuidados seguros, económicos y oportunos de cirugía y anestesia corresponde a la densidad de fuerza laboral de especialistas quirúrgicos ${ }^{1}$. Cirugía Global 2030, una iniciativa global de Lancet Comission, para la administración, financiamiento y provisión de cuidados quirúrgicos, alrededor de los cuales puede realizarse una alianza global para alcanzar equidad en salud, estima que los países deben contar con al menos 20 cirujanos, anestesiólogos y obstetras por cada 100.000 habitantes ${ }^{1}$. Para alcanzar esta meta se requieren esfuerzos en diferentes direcciones. Son necesarios los registros en materia de fuerza laboral, los datos de instituciones de entrenamiento y licencias para ejercer la cirugía, que permitan realizar los ajustes necesarios y proyecciones futuras ${ }^{2}$. También es necesario robustecer la infraestructura educativa, la cooperación y la cirugía global, a la vez que se reduce la deserción de los residentes (estimada globalmente en 20\%) y se mejoran las condiciones de práctica profesio$\mathrm{nal}^{2-4}$. Finalmente, es necesario atraer y cautivar a los estudiantes de medicina para que opten por una carrera en cirugía ${ }^{5,6}$.

No obstante, la transformación acelerada de la especialidad a nivel global, así como factores de orden cultural, económico y político amenazan esta iniciativa ${ }^{7}$. Inclusive, el número de cirujanos ha disminuido globalmente ${ }^{8}$. En el campo de la educación médica, en particular, existen factores que explican el menor interés de los estudiantes hacia la especialidad y el subsecuente impacto negativo en la formación del recurso humano. La excesiva carga de trabajo, los desafíos del entrenamiento (incluido el acoso, maltrato y discriminación) y el pobre equilibrio entre el trabajo y la familia son algunos factores $^{9-12}$. Así mismo, el énfasis creciente de los cirujanos en los aspectos técnicos, individualidad y rendimiento, debido a presiones por eficiencia administrativa y resultados, y las demandas legales se han convertido en barreras para bienestar profesional disminuyendo la atracción por la especiali$\mathrm{dad}^{6,12,13}$. Estos cambios son, a la vez, la principal fuente de desgaste profesional y de pobre calidad de vida que muchos estudiantes pretenden evitar en su carrera profesional ${ }^{14}$. Los desafíos son aún más exigentes en materia de género. Las mujeres deben ser innovadoras al momento de combinar su rol como mujer y cirujano, situación que exige mayores esfuerzos, modelos y trayectorias paradigmáticas para quienes aspiran a esta carrera ${ }^{15-17}$.
Frente a estas transformaciones, existe un llamado permanentemente a evaluar las condiciones de la práctica profesional y los factores que favorecen y/o limitan la intención de optar por una carrera en cirugía en diversos contextos ${ }^{1,2,18}$. Esta evaluación es importante porque la dinámica de la práctica profesional, los desafíos epidemiológicos y demográficos, y los retos educativos son inseparables de la planeación del recurso humano en salud. En el presente estudio pretendemos evaluar la intención que tienen los estudiantes de medicina de optar por una carrera en cirugía en un contexto de inequidad y vulnerabilidad en materia de salud, y de grandes retos en materia de educación médica, e identificar los factores asociados con esta intención.

\section{Materiales y Método}

\section{Contexto y participantes}

Este estudio transversal fue realizado en Colombia. Según cifras del Ministerio de Salud de Colombia, el país cuenta con 1.843 cirujanos $^{19}$. $\mathrm{La}$ cobertura nacional aproximada es de 3,68 cirujanos/100.000 habitantes. Anualmente, se ofertan 400 posiciones de residencia en 20 programas (cuatro años de duración $)^{20}$. Los residentes de cirugía deben pagar a las universidades para realizar la especialización (el costo promedio de la matrícula por semestre oscila entre 3.000-5.000 USD) y no reciben salario. La reglamentación nacional de trabajo es de $66 \mathrm{~h}$ semanales.

Los aspirantes a la especialización provienen, por lo general, de las 58 facultades de medicina activas en Colombia (28 con Acreditación de Alta Calidad $)^{21}$. Trece están ubicadas en Bogotá y sus alrededores (Región Sabana Centro). La duración promedio de la carrera es de seis años, incluyendo uno de internado. Posteriormente, los graduados realizan un año de servicio social obligatorio. Una vez finalizado, los médicos generales pueden aplicar a la residencia de su preferencia en instituciones universitarias. No existe un examen de admisión nacional por lo que cada universidad cuenta con sus propios criterios de selección.

Para la presente investigación invitamos a 500 estudiantes de medicina, provenientes de diez de las trece universidades acreditadas ubicadas en Bogotá y sus alrededores (Región Sabana Centro), quienes se encontraban finalizando su rotación por cirugía general en el cuarto año de carrera.

\section{Mediciones}

En el presente estudio, el concepto de "carrera" profesional representa un proceso de decisión ac- 
tivo del individuo influenciado por la transformación dinámica de las organizaciones en respuesta a cambios acelerados en el ambiente laboral por la competitividad, globalización e incursión tecnológica $^{22}$. Nuestra fundamentación teórica evita posturas tradicionales de carrera como vocación $u$ ocupación en la que un individuo encaja, como la búsqueda de una organización o como una secuencia de roles organizacionales y estatus. En este sentido, el concepto de carrera, está determinado por un comportamiento intencionado que se orienta por la ponderación continua y racional del individuo sobre el ambiente a partir de sus preferencias ${ }^{22}$. Con base en estos argumentos, inicialmente realizamos una revisión de la literatura para identificar los factores que influencian la intención de realizar una carrera en cirugía general. A continuación, desarrollamos un cuestionario de 25 ítems para evaluar la asociación entre los factores identificados y la intención de los estudiantes de medicina hacia la especialidad. La revisión de la literatura, y una prueba piloto entre 30 internos, permitieron asegurar la validez de contenido y apariencia del cuestionario.

El cuestionario está compuesto por ocho dominios: 1) influencia de género en la especialidad; 2) identificación en el estilo de vida quirúrgico (influencias de la especialidad en la vida personal, social y familiar); 3 ) identificación con modelos (profesores o residentes que ejercen un modelo positivo sobre el estudiante); 4) atributos personales (rasgos de personalidad); 5) recompensa futura (retribución futura material o no material, por ejemplo dinero); 6) prestigio de la especialidad; 7) identificación con la práctica profesional del cirujano (dinámica de la práctica profesional en el sitio de trabajo) y 8) dinámica del posgrado. Los ítems fueron calificados mediante una escala de Likert de 5-puntos: 1 (Totalmente en desacuerdo); 5 (Totalmente de acuerdo). La intención fue evaluada mediante el siguiente ítem: "He considerado realizar mi especialización en cirugía general". En consenso, definimos una alta intención si la respuesta fue $\geq 4,0$, moderada entre 3,1-3,9 y baja $\leq 3,0$. Las mediciones fueron realizadas en octubre de 2018, y el cuestionario fue administrado en papel. El cuestionario fue diligenciado voluntariamente por los participantes, de forma confidencial y anónima, tras recibir una explicación escrita sobre el alcance de la investigación y uso posterior de la información, y obtener un consentimiento verbal.

\section{Análisis estadístico}

Inicialmente calculamos los puntajes globales para cada uno de los ítems del cuestionario y do- minios utilizando medias y desviaciones estándar (DE). Las correlaciones (Pearson) fueron calculadas para todas las combinaciones posibles de las variables. El tamaño del efecto para cada correlación fue interpretado así: $r= \pm 0,10$ (pequeño), $r= \pm 0,30$ (mediano), $r= \pm 0,50$ (grande) $^{23}$. Anticipamos una muestra de 194 participantes para determinar si los coeficientes de correlación entre las variables difieren de 0 (valor mínimo $\mathrm{r}=0,20$ ) con un error Tipo $\mathrm{I}=0,05$ y Tipo $\mathrm{II}=0,20$. La consistencia interna del cuestionario se estableció mediante el coeficiente $\alpha$ de Cronbach (satisfactorio si $>0,70)^{24}$.

Posteriormente, para evaluar las asociaciones entre la intención (variable dependiente) con la influencia de género en la especialidad, identificación con el estilo de vida quirúrgico, la identificación con modelos, los atributos personales, la recompensa futura, el prestigio de la especialidad, identificación con la práctica profesional del cirujano y la dinámica del posgrado (variables independientes), realizamos un análisis de regresión lineal. Los resultados de regresión se expresaron mediante coeficientes no estandarizados (b) y estandarizados $(\beta)$, errores estándar e intervalos de confianza del $95 \%$ ( $p<0,05)$. Igualmente, calculamos el coeficiente de determinación múltiple $\left(\mathrm{R}^{2}\right)$, como indicador de la varianza ocupada por cada una de las variables independientes sobre la dependiente $(\mathrm{p}<0,05)$. El tamaño del efecto representado por el valor del $\mathrm{R}^{2}$ fue interpretado así: $\mathrm{R}^{2}=0,02$ (pequeño), $\mathrm{R}^{2}=0,13$ (medio) y $\mathrm{R}^{2}=0,26$ (alto) ${ }^{23}$. Finalmente, calculamos el Factor de Inflación de Varianza (FIV) con el fin de establecer si la relación entre las variables incluidas en el modelo estuvo afectada por multicolineali$\mathrm{dad}(\mathrm{VIF} \geq 10 \mathrm{o} \text { índice de tolerancia } 1 / \mathrm{VIF} \leq 0,3)^{23}$.

\section{Resultados}

Un total de 252 estudiantes (65,87\% mujeres), denotando una tasa de respuesta de $50 \%$, fueron incluidos en el análisis. La edad promedio de los participantes fue 22,20 \pm 1,92 (19-35) años. El promedio global de la intención de optar por una carrera en cirugía fue 3,15 $\pm 1,34$ (IC 95\% 2,99-3,32). Este promedio varío según el género (Hombres $=3,40 \pm$ 1,2 ; Mujeres $=3,03 \pm 1,36, \mathrm{p}<0,01$ ).

Los puntajes de las variables independientes variaron desde 2,43 $\pm 1,23$ (influencia de género en la especialidad) hasta $3,82 \pm 0,93$ (recompensa futura). Los valores de las correlaciones (Pearson) variaron desde 0,19 (identificación con modelos-influencia de género en la especialidad), hasta 0,65 (identificación con la práctica profesional de cirujano- 
Tabla 1. Estadísticas descriptivas y correlaciones de las variables incluidas en el estudio

\begin{tabular}{|c|c|c|c|c|c|c|c|c|c|c|c|}
\hline Variables & Media & DE & 1 & 2 & 3 & 4 & 5 & 6 & 7 & 8 & 9 \\
\hline Intención de optar por una carrera en cirugía $(1)^{*}$ & 3,15 & 1,34 & 1 & & & & & & & & \\
\hline Influencia de género en la especialidad (2)* & 2,43 & 1,23 & 0,20 & 1 & & & & & & & \\
\hline Identificación con el estilo de vida quirúrgico (3)* & 2,87 & 0,95 & 0,62 & 0,30 & 1 & & & & & & \\
\hline Identificación con modelos (4)* & 3,09 & 1,37 & 0,54 & 0,19 & 0,50 & 1 & & & & & \\
\hline Prestigio de la especialidad $(7)^{*}$ & 3,38 & 1,24 & 0,52 & 0,29 & 0,62 & 0,42 & 0,57 & 0,49 & 1 & & \\
\hline Identificación con la práctica profesional del cirujano $(8)^{*}$ & 3,57 & 0,90 & 0,51 & 0,34 & 0,59 & 0,43 & 0,49 & 0,65 & 0,59 & 1 & \\
\hline Dinámica del posgrado (9)* & 2,79 & 0,78 & 0,48 & 0,34 & 0,59 & 0,45 & 0,49 & 0,43 & 0,64 & 0,54 & 1 \\
\hline
\end{tabular}

*Rango 0-5, DE: Desviación estándar. Todas las correlaciones (Pearson) fueron estadísticamente significativas ( $\mathrm{p}<0,05)$.

Tabla 2. Resultados de la regresión lineal evaluando las asociaciones entre las variables independientes con la intención de los estudiantes de optar por una carrera en cirugía

\begin{tabular}{|c|c|c|c|c|c|}
\hline Variables & b & $\mathbf{E E}$ & $\mathbf{p}$ & IC $95 \%$ & $\boldsymbol{\beta}$ \\
\hline Influencia de género en la especialidad & $-0,05$ & 0,05 & & $-0,15-0,05$ & $-0,04$ \\
\hline Identificación con el estilo de vida quirúrgico & 0,35 & 0,09 & $*$ & $0,17-0,54$ & 0,25 \\
\hline Identificación con modelos & 0,18 & 0,05 & $*$ & $0,07-0,29$ & 0,18 \\
\hline Atributos personales & 0,38 & 0,09 & $*$ & $0,19-0,57$ & 0,25 \\
\hline Identificación con la práctica profesional del cirujano & 0,25 & 0,10 & $*$ & $0,04-0,45$ & 0,16 \\
\hline Dinámica del posgrado & 0,05 & 0,10 & & $-0,15-0,27$ & 0,03 \\
\hline
\end{tabular}

${ }^{*} \mathrm{p}<0,05$. b: coeficiente de regresión no estandarizado, $\beta$ : coeficiente de regresión estandarizado, IC: intervalo de confianza, EE: error estándar

recompensa futura). Todas las correlaciones fueron estadísticamente significativas $(\mathrm{p}<0,05)$. Todas las correlaciones, media y desviaciones de cada variable se encuentran en la Tabla 1. El coeficiente $\alpha$ de Cronbach del cuestionario fue 0,91 .

En la regresión lineal la combinación de las variables independientes ocupó un 52\% de la varianza de la intención, denotando un alto tamaño del efecto $\mathrm{R}^{2}=0,52(\mathrm{~F}=33,10 \mathrm{p}<0,001)$. Los coeficientes estandarizados para las variables independientes significativos $(p<0,05)$ fueron: a) identificación con el estilo de vida quirúrgico, $\beta=0,25$; b) identificación con modelos, $\beta=0,18$; c) atributos personales, $\beta=0,25 ; \mathrm{y}$ d) identificación con la práctica profesional del cirujano, $\beta=0,16$. Los VIF para cada una de las variables oscilaron entre 1,20-2,50. Así mismo, los índices de tolerancia (1/VIF) variaron entre 0,40 0,83 descartando multicolinealidad. El diagnóstico de la regresión se encuentra en la Tabla 2.

\section{Discusión}

En el presente estudio evaluamos la intención (proxi de la conducta futura) de estudiantes de medicina colombianos de optar por una carrera en cirugía. Esta evaluación se ajustó a nuestra perspectiva teórica de "carrera" como un proceso de decisión racional activo, y no estático como en las perspectivas teóricas tradicionales ${ }^{22}$. Encontramos una moderada intención, asociada positivamente con la identificación con el estilo de vida quirúrgico, la 
identificación con modelos, los atributos personales y la identificación con la práctica profesional del cirujano. No encontramos asociación significativa, positiva o negativa, con el género (mujeres en la especialidad), prestigio, dinámica del posgrado ni recompensa futura.

Nuestros hallazgos tienen varias explicaciones. La intención de "convertirse" en cirujano denota la voluntad individual para desarrollar una identidad profesional en un complejo ambiente de práctica, cultura y normas, que implican un estilo de vida y práctica profesional exigentes ${ }^{25-27}$. Creemos que el estilo de vida y la práctica son factores que continúan siendo atractivos para quienes quieren optar por la especialidad, y evocan aspectos emocionales, pasionales y relacionados con los valores de la profesión ${ }^{28,29}$. Nuestros resultados indican que los atributos personales son fundamentales para lograr un buen ajuste con esta cultura, así como con las demandas del ambiente. Estos hallazgos han sido reconocidos en estudios preliminares que enfatizan la importancia de perfiles de personalidad orientados a la resolución de problemas, toma de decisiones, liderazgo y proactividad, entre otros ${ }^{6,30,31}$. En este esfuerzo resaltamos la relevancia de los modelos en la especialidad para facilitar la decisión de los estudiantes. Este factor es crucial, pues aquellos profesores, residentes o supervisores capaces de influir, positiva o negativamente, en el descubrimiento de la cirugía como opción de vida, permiten que los estudiantes puedan negociar más fácilmente el currículo oculto de la profesión, mejorar las percepciones sobre el estilo de vida y la práctica, y disipar los estereotipos negativos ${ }^{32-36}$ Este hallazgo, es congruente con estudios preliminares realizados en Colombia ${ }^{37}$.

Respecto a otros factores, la influencia de género en la especialidad no demostró asociación significativa. Consideramos que, a diferencia de otros contextos, durante los últimos años existe un aumento progresivo en el número de mujeres en cirugía en Colombia, lo cual podría indicar la apertura de los programas y de la comunidad quirúrgica hacia un amplio rango de aspirantes. Este fenómeno también puede asociarse con el mayor número de mujeres en la carrera de medicina en el país, estimado globalmente en el $58,2 \% \%^{21}(65,87 \%$ en el presente estudio). Anticipamos un mayor número de mujeres en la cirugía colombiana en los próximos años. Igualmente, el prestigio, la dinámica del posgrado y la recompensa futura no demostraron asociación. Estos hallazgos pueden explicarse por la transformación acelerada de la especialidad en Colombia, por el creciente número de opciones de desarrollo profesional y opciones laborales (especialidades, maestrías y doctorados en diversas disciplinas) y por las reglamentaciones progresivas implementadas para mejorar el bienestar de los residentes (por ejemplo, restricción en el número de horas laborales). Llamativamente, en comparación con estudios preliminares, encontramos que el prestigio y las oportunidades futuras, consideradas como factores con asociación positiva para la elección en Colombia $^{37}$, hoy en día cobran menor relevancia. No obstante, el presente estudio dista de explicaciones específicas a la falta de asociación actual de estos factores, así como a la variación de estas preferencias en los últimos años. Invitamos a realizar nuevos estudios al respecto.

Este estudio tiene fortalezas y limitaciones. Dentro de las primeras se encuentra la medición de percepciones bajo fundamentos teóricos y factores relevantes identificados en la literatura. El estudio cuenta con un instrumento válido (contenido y apariencia) que permite acercarnos a la exploración de un constructo complejo. Igualmente, cuenta con un número significativo de participantes provenientes de un amplio rango de instituciones, que permitió realizar inferencias y obtener conclusiones cercanas a la realidad. No obstante, el instrumento puede mejorarse, en particular si se determina su validez de constructo. También consideramos que el estudio evaluó participantes provenientes de una sola región del territorio colombiano, de gran influencia y tradición en la educación médica del país, lo cual puede limitar su generalización a otras regiones y contextos. Finalmente, la medición se realizó en un momento específico de la carrera (cuarto año). Es importante anotar que a este nivel algunos estudiantes no han realizado otras rotaciones clínicas, lo cual puede afectar sus preferencias. Invitamos a realizar investigaciones con seguimiento longitudinal y mediciones en diferentes momentos de la carrera.

El estudio ofrece oportunidades para la práctica y opciones para la investigación futura aparte de las ya mencionadas. En la práctica permite identificar el rol de la mentoría y motivación estudiantil de los supervisores y residentes, desde las rotaciones de cirugía. Igualmente ofrece oportunidades para mejorar la exposición temprana de estudiantes de medicina al campo de la cirugía general, disipar las percepciones negativas sobre la cirugía general y abrir al escrutinio público los problemas de calidad de vida resultantes del ejercicio profesional. La creación de espacios académicos que ofrezcan oportunidades de participación de los estudiantes (foros, congresos y seminarios), desarrollo de sus facetas de liderazgo y discusión, pueden contribuir a estos propósitos. 
Consideramos que se requieren estudios nacionales y regionales sobre estos temas, así como comparaciones con otros contextos. Igualmente, se deben explorar a profundidad las asociaciones positivas y negativas del ambiente con la intención de realizar una carrera quirúrgica. Los estudios cualitativos a este respecto son bienvenidos.

En conclusión, hemos evaluado de forma transversal la intención que tienen los estudiantes de medicina colombianos de optar por una carrera quirúrgica y sus factores asociados en un contexto de aceleradas transformaciones en la educación médica, ofreciendo oportunidades para la práctica e identificando vacíos de conocimiento que pueden explorarse en nuevos estudios.

\section{Responsabilidades éticas}

Protección de personas y animales. Los autores declaran que para esta investigación no se han realizado experimentos en seres humanos ni en animales.

Confidencialidad de los datos. Los autores declaran que en este artículo no aparecen datos de pacientes.

Financiación: Facultad de Medicina, Universidad de la Sabana, Chía (Colombia).

Conflicto de Intereses: Ninguno.

\section{Referencias}

1. Meara JG, Leather AJM, Hagander L, Alkire BC, Alonso N, Ameh EA, et al. Global Surgery 2030: Evidence and solutions for achieving health, welfare, and economic development Edgar Rodas, MD died March 2, 2015; We dedicate our report to him. [Internet]. Vol. 213, American Journal of Obstetrics and Gynecology. 2015 [citado el 26 de mayo de 2018]. p. 338-40. Available from: http://www.ncbi.nlm.nih.gov/ pubmed/25985722.

2. Haider A, Scott JW, Gause CD, Meheš M, Hsiung G, Prelvukaj A, et al. Development of a Unifying Target and Consensus Indicators for Global Surgical Systems Strengthening: Proposed by the Global Alliance for Surgery, Obstetric, Trauma, and Anaesthesia Care (The G4 Alliance). World J Surg [Internet]. 2017 [citado el 21 de noviembre de 2018];41(10):2426-34. Available from: http://www.ncbi.nlm.nih. gov/pubmed/28508237.

3. Debas HT, Donkor P, Gawande A, Jamison DT, Kruk ME, Mock CN Essential Surgery [Internet]. Essential Surgery: Disease Control Priorities, Third Edition (Volume 1). The International Bank for Reconstruction and Development/The World Bank; 2015 [citado el 21 de noviembre de 2018]. Available from: http://www.ncbi.nlm.nih. gov/pubmed/26740991.

4. Khoushhal Z, Hussain MA, Greco E, Mamdani M, Verma S, Rotstein O, et al.
Prevalence and causes of attrition among surgical residents a systematic review and meta-analysis. In: JAMA Surgery [Internet]. 2017 [citado el 25 de mayo de 2018]. p. 265-72. Available from: http://archsurg.jamanetwork.com/article. aspx?doi=10.1001/jamasurg.2016.4086

5. Scally CP, Minter RM. Medical School Training for the Surgeon. Surg Clin North Am [Internet]. 2016 [citado el 21 de noviembre de 2018];96(1):1-13. Available from: http://www.ncbi.nlm.nih.gov/ pubmed/26612015.

6. Schmidt LE, Cooper CA, Guo WA. Factors influencing US medical students' decision to pursue surgery. J Surg Res [Internet]. 2016 [citado el 21 de noviembre de 2018];203(1):64-74. Available from: http://www.ncbi.nlm.nih. gov/pubmed/27338536.

7. Saluja S, Silverstein A, Mukhopadhyay S, Lin Y, Raykar N, Keshavjee S, et al. Using the Consolidated Framework for Implementation Research to implement and evaluate national surgical planning. BMJ Glob Heal [Internet]. 2017 [citado el 21 de noviembre de 2018];2(2):e000269. Available from: http://www.ncbi.nlm.nih. gov/pubmed/29225930

8. Are C. Workforce Needs and Demands in Surgery. Surg Clin North Am [Internet]. 2016 [citado el 23 de noviembre de 2018];96(1):95-113. Available from: https://linkinghub.elsevier.com/retrieve/ pii/S0039610915001632.

9. Halim UA, Riding DM. Systematic review of the prevalence, impact and mitigating strategies for bullying, undermining behaviour and harassment in the surgical workplace. Br J Surg [Internet]. 2018 [citado el 21 de noviembre de 2018];105(11):1390-7. Available from: http://www.ncbi.nlm.nih.gov/ pubmed/30007001.

10. Seelandt JC, Kaderli RM, Tschan F, Businger AP. The surgeon's perspective: promoting and discouraging factors for choosing a career in surgery as perceived by surgeons. Burney RE, editor. PLoS One [Internet]. 2014 [citado el 21 de noviembre de 2018];9(7):e102756. Available from: https://dx.plos. org/10.1371/journal.pone.0102756.

11. Borracci RA, Ferraina P, Arribalzaga EB, Poveda Camargo RL. Elegir a la cirugía como especialidad: Opiniones de los estudiantes de la Universidad de Buenos Aires sobre la cirugía y los cirujanos. Cirugía Española [Internet]. 2014 [citado el 21 de noviembre de 2018];92(9):619-

24. Available from: http://www.ncbi.nlm. nih.gov/pubmed/24237853.

12. Pointer DT, Freeman MD, Korndorffer JR, Meade PC, Jaffe BM, Slakey DP. Choosing Surgery: Identifying Factors Leading to Increased General Surgery Matriculation Rate. Am Surg [Internet]. 2017 [citado el 21 de noviembre de 2018];83(3):290-5. Available from: http://www.ncbi.nlm.nih.gov/ pubmed/28316314.

13. Orri M, Farges O, Clavien P-A, Barkun J, Revah-Lévy A. Being a Surgeon-The Myth and the Reality. Ann Surg [Internet]. 
2014 [citado el 21 de noviembre de 2018];260(5):721-9. Available from: http://www.ncbi.nlm.nih.gov/ pubmed/25379843.

14. Grigg M, Arora M, Diwan AD. Australian medical students and their choice of surgery as a career: a review. ANZ J Surg [Internet]. 2014 [citado el 21 de noviembre de 2018];84(9):653-5. Available from: http://www.ncbi.nlm.nih. gov/pubmed/24103025.

15. Hill E, Solomon Y, Dornan T, Stalmeijer R. "You become a man in a man's world": is there discursive space for women in surgery? Med Educ [Internet]. 2015 [citado el 21 de noviembre de 2018];49(12):1207-18. Available from: http://doi.wiley.com/10.1111/medu. 12818 .

16. Hill EJR, Giles JA. Career decisions and gender: the illusion of choice? Perspect Med Educ [Internet]. 2014 [citado el 21 de noviembre de 2018];3(3):151-4. Available from: http://link.springer.com/10.1007/ s40037-014-0128-x.

17. Hill E, Vaughan S. The only girl in the room: how paradigmatic trajectories deter female students from surgical careers. Med Educ [Internet]. 2013 [citado el 21 de noviembre de 2018];47(6):54756. Available from: http://doi.wiley. com/10.1111/medu.12134.

18. Borracci RA, Ferraina P, Arribalzaga EB, Poveda Camargo RL. Elegir a la cirugía como especialidad: Opiniones de los estudiantes de la Universidad de Buenos Aires sobre la cirugía y los cirujanos. Cirugía Española [Internet]. 2014 [citado el 2 de febrero de 2019];92(9):619-24. Available from: https://inkinghub.elsevier.com/retrieve/ pii/S0009739X1300331X.

19. Ministerio de Salud y Protección Social (Colombia). Aproximaciones a la estimación de la oferta y la demanda de médicos especialistas en Colombia, 2015-2030. 2017 [citado el 26 de mayo de 2018];1-23. Available from: https://www.minsalud.gov.co/sites/rid/ Lists/BibliotecaDigital/RIDE/VS/TH/ Especialistas-md-oths.pdf.

20. Dominguez LC, Stassen L, de Grave W, Sanabria A, Alfonso E, Dolmans D. Taking control: Is job crafting related to the intention to leave surgical training? Koniaris LG, editor. PLoS One [Internet]. 2018 Jun 1 [citado el 2 de junio de 2018];13(6):e0197276. Available from: http://www.ncbi.nlm.nih.gov/ pubmed/29856750.

21. Asociación Colombiana de Facultades de Medicina (ASCOFAME). Estadísticas básicas de la educación médica en Colombia 2016 [Internet]. Bogota; 2017 [citado el 21 de noviembre de 2018]. Available from: https://snies. mineducacion.gov.co

22. Inkson K. Contemporary Conceptualizations of Career. APA Handb Career Interv. 2015;1:21-42.

23. Hatcher L. Advanced statistics in research: reading, understanding, and writing up data analysis results. ShadowFinch Media, LLC; 2013. $632 \mathrm{p}$.

24. Cronbach LJ, Warrington WG. Timelimit tests: Estimating their reliability and degree of speeding. Psychometrika [Internet]. 1951 [citado el 16 de julio de 2018];16(2):167-88. Available from: http://link.springer.com/10.1007/ BF02289113.

25. Lovell B. How to build a resilient surgeon: communities, cultures and complexity. Med Educ [Internet]. 2016 [citado el 23 de noviembre de 2018];50(8):8002. Available from: http://doi.wiley. com/10.1111/medu. 13081 .

26. Patel P, Martimianakis MA, Zilbert NR, Mui C, Hammond Mobilio M, Kitto S, et al. Fake It 'Til You Make It: Pressures to Measure Up in Surgical Training. Acad Med [Internet]. 2018 May [citado el 23 de noviembre de 2018];93(5):769-74. Available from: http://insights.ovid.com/cr ossref?an=00001888-201805000-00042.

27. Cahan MA, Starr S, Larkin AC, Litwin DEM, Sullivan KM, Quirk ME.

Transforming the culture of surgical education: promoting teacher identity through human factors training. Arch Surg [Internet]. 2011 [citado el 23 de noviembre de 2018];146(7):830-4. Available from: http://archsurg.jamanetwork.com/article. aspx?doi=10.1001/archsurg.2011.157.

28. Cope A, Bezemer J, Mavroveli S, Kneebone R. What Attitudes and Values Are Incorporated Into Self as Part of Professional Identity Construction When Becoming a Surgeon? Acad Med [Internet]. 2017 Apr [citado el 23 de noviembre de 2018];92(4):544-9. Available from: http://insights.ovid.com/cr ossref?an=00001888-201704000-00047.
29. Singletary SE. A fire in our hearts: passion and the art of surgery. Ann Surg Oncol [Internet]. 2010 [citado el 23 de noviembre de 2018];17(2):364-70. Available from: http://www.springerlink. com/index/10.1245/s10434-009-0732-x.

30. Burgos CM, Josephson A. Gender differences in the learning and teaching of surgery: a literature review. Int J Med Educ [Internet]. 2014 [citado el 23 de noviembre de 2018];5:110-24. Available from: http://www.ijme.net/archive/5/ gender-differences-in-learning-andteaching-of-surgery/

31. Preece RA, Cope AC. Are Surgeons Born or Made? A Comparison of Personality Traits and Learning Styles Between Surgical Trainees and Medical Students. J Surg Educ [Internet]. 2016 [citado el 23 de noviembre de 2018];73(5):768-73. Available from: https://linkinghub.elsevier.com/retrieve/ pii/S193172041630006X.

32. Hill E, Bowman K, Stalmeijer R, Hart J. You've got to know the rules to play the game: how medical students negotiate the hidden curriculum of surgical careers. Med Educ [Internet]. 2014 [citado el 23 de noviembre de 2018];48(9):88494. Available from: http://doi.wiley. com/10.1111/medu.12488.

33. Hill EJR, Bowman KA, Stalmeijer RE, Solomon Y, Dornan T. Can I cut it? Medical students' perceptions of surgeons and surgical careers. Am J Surg [Internet]. 2014 [citado el 23 de noviembre de 2018];208(5):860-7. Available from: https://linkinghub.elsevier.com/retrieve/ pii/S0002961014002803.

34. Neumayer L, Kaiser S, Anderson K, Barney L, Curet M, Jacobs D, et al. Perceptions of women medical students and their influence on career choice. Am J Surg [Internet]. 2002 [citado el 23 de noviembre de 2018];183(2):146-50. Available from: http://www.ncbi.nlm.nih. gov/pubmed/11918878.

35. Sutton PA, Mason J, Vimalachandran D, McNally S. Attitudes, motivators, and barriers to a career in surgery: a national study of U.K. undergraduate medical students. J Surg Educ [Internet]. 2014 [citado el 23 de noviembre de 2018];71(5):662-7. Available from: https://linkinghub.elsevier.com/retrieve/ pii/S1931720414000786. 
ARTÍCULO ORIGINAL

36. Cochran A, Paukert JL, Neumayer LA. Does a general surgery clerkship influence student perceptions of surgeons and surgical careers? Surgery [Internet]. 2003 [citado el 23 de noviembre de
2018];134(2):153-7. Available from: http://linkinghub.elsevier.com/retrieve/pii/ S0039606003500599.

37. Domínguez LC, Sanabria ÁE, Vega NV, Espitia EL, Cabrera EY, Osorio C, et al. Factores que influencian la elección de una carrera quirúrgica: análisis multiinstitucional global desde una perspectiva académica en Bogotá. Rev Colomb Cirugía 2013;28:275-81. 